Aim of the study was to analyze the outcome of treatment and factors predicting results of sorafenib therapy in inoperable/metastatic CD117-positive GIST patients after failure on imatinib and sunitinib.

Material and methods: We identified 60 consecutive patients (40 men, 20 women) with advanced inoperable/ metastatic GIST after failure on at least imatinib and sunitinib treated in one sarcoma center with sorafenib at initial dose $2 \times 400 \mathrm{mg}$ daily in 2007-2015 (in 56 cases it was $3^{\text {rd }}$ line therapy). Median follow-up time was 39 months.

Results: One year progression-free survival (PFS; calculated from the date of the start of sorafenib to disease progression) rate was $23 \%$ and median PFS $=7.7$ months. The median overall survival (OS) was 13.5 months calculated from sorafenib start (1-year OS rate $=57 \%)$ and 7 years from imatinib start. Three patients $(5 \%)$ had objective partial responses to therapy, 31 patients $(52 \%)$ had stabilization of disease $>4$ months. Primary tumor mutational status was known in 43 cases $(73 \%)$, but we have not identified the differences in PFS between tumors carrying different KIT/PDGFRA mutations. The most common adverse events were: diarrhoea, hand and foot syndrome, fatigue, loss of weight and skin reactions; grade 3-5 toxicity occurred in $35 \%$ of patients. 23 patients required sorafenib dose reductions due to AEs.

Conclusions: We confirmed that many advanced GIST patients benefit from sorafenib therapy after imatinib/sunitinib failure with OS > 1 year.

Key words: gastrointestinal stromal tumor, sorafenib, metastases, therapy.

Contemp Oncol (Pozn) 2017; 21 (4): 285-289 DOI: https://doi.org/10.5114/wo.2017.72393

\section{The analysis of the long-term outcomes of sorafenib therapy in routine practice in imatinib and sunitinib resistant gastrointestinal stromal tumors (GIST)*}

Piotr Rutkowski, Beata Jagielska, Jolanta Andrzejuk, Elzbieta Bylina, Iwona Lugowska, Tomasz Switaj, Hanna Kosela-Paterczyk, Katarzyna Kozak, Slawomir Falkowski, Anna Klimczak

Maria Sklodowska-Curie Institute - Oncology Center, Warsaw, Poland

*The preliminary data of this study were presented during Connective Tissue Oncology Society Meeting, 2015 in Salt Lake City, UT.

\section{Introduction}

In recent years, the management of gastrointestinal stromal tumors (GIST) has changed with the understanding molecular mechanisms of their pathogenesis. The detection of activating, somatic, mutually exclusive mutations of two genes, KIT and PDGFRA (platelet-derived growth factor receptor- $\alpha$ ) in the majority of these tumors, has led to the development of targeted therapy with tyrosine kinase inhibitors, as imatinib and sunitinib $[1,2]$. The introduction of imatinib mesylate into clinical practice has revolutionized the therapy of advanced GIST with 4-5-fold increase in overall survival of patients as compared to historical data and this drug became first line standard of care in metastatic/unresectable GIST [3, 4]. However, the median progression-free survival (PFS) of imatinib-treated patients is 2-3 years $[3,4]$. A second-generation tyrosine kinase inhibitor - sunitinib with a broader spectrum of action, is effective in improving PFS in patients with GIST who are resistant or intolerant to imatinib (with median PFS 6-8 months) and it is the only second line therapy for this clinical indication [5]. Until recently, in cases of advanced progression, there were no standard methods of treatment and the inclusion of patients into clinical trials of new drugs was recommended. Regorafenib, a new multiple kinases inhibitor, is indicated as $3^{\text {rd }}$-line treatment in patients with locally advanced, unresectable or metastatic GIST who have been previously treated with imatinib and sunitinib [6, 7], but the median PFS observed in phase III trial with regorafenib was 4.8 months. It implies that the options in the $3^{\text {rd }} / 4^{\text {th }}$ line of therapy are still very limited. Regorafenib is closely related to sorafenib (differing only by the addition of a fluorine atom to the center phenyl ring), but it has a distinct biochemical profile with other activity than sorafenib against VEGFR-2, PDGFRB, FGFR-1, KIT and TIE-2 [8, 9]. Nevertheless, in Poland sorafenib is reimbursed by health insurance in inoperable/metastatic GIST patients after failure of imatinib and sunitinib, based on the results of phase II clinical trials [10-12]. Sorafenib is also a recommended treatment option based on National Comprehensive Cancer Network (NCCN) guidelines [13]. The aim of the study was to analyze the outcome of treatment and factors predicting results of sorafenib therapy in inoperable/metastatic CD117-positive GIST patients after failure on imatinib and sunitinib treated in routine practice based on our hypothesis that sorafenib can be a substitute of regorafenib in the $3^{\text {rd }}$ line therapy of advanced GIST. 


\section{Material and methods}

\section{Patients}

We analyzed data of 60 consecutive patients treated with sorafenib due of inoperable and/or metastatic CD117-positive GIST, who started treatment between November 2007 and August 2015 in one reference sarcoma center. All patients met the following criteria for sorafenib treatment: 1) histological diagnosis of GIST, confirmed by CD117-immunopositivity, 2) metastatic and/or inoperable lesions after failure on at least prior treatment with imatinib and sunitinib (in analyzed series all patients had confirmed progressive disease on both lines of therapy), 3) measurable disease on computed tomography (CT) scans, 4) WHO performance status $\leq 3,5$ ) no concomitant oncological therapy for disease, 6) adequate renal, cardiac and liver function.

The study had been approved by the local Bio-Ethics Committee according to Good Clinical Practice Guidelines (approvals from Bio-Ethics Committees from Maria Sklodowska-Curie Institute - Oncology Center, Warsaw KB/9/2011 and approval for Polish Clinical GIST Registry by Internal Review Board 119/2002). All patients has signed informed consent for sorafenib treatment and using their data for research purposes. Patients did not undergo any further selection.

All patients were treated with sorafenib with initial dose of $400 \mathrm{mg}$ bid, however the dosing could be reduced (to $600 \mathrm{mg}$ or $400 \mathrm{mg}$ daily) or delayed to optimize the benefit-risk profile according to decision of treating physician. The treatment was continued until confirmed progression of the disease or unacceptable toxicity. All patients were followed carefully with median follow-up time of 26 months (range: 4-83 months). The objective response of GIST to sorafenib therapy was evaluated with serial CT examinations (performed every $2-3$ months), ac-

Table 1. Characteristics of 60 patients treated with sorafenib due to advanced GIST

\begin{tabular}{|c|c|c|}
\hline \multicolumn{2}{|c|}{ Clinicopathological features } & \multirow{2}{*}{$\begin{array}{c}\text { No. of } \\
\text { patients } \\
(\%) \\
59(22-79)\end{array}$} \\
\hline $\begin{array}{l}\text { Age [years] at the } \\
\text { start of therapy } \\
\text { with sorafenib }\end{array}$ & Median (range) & \\
\hline Gender & $\begin{array}{l}\text { Female } \\
\text { Male }\end{array}$ & $\begin{array}{l}20(33) \\
40(67)\end{array}$ \\
\hline $\begin{array}{l}\text { Primary tumor } \\
\text { site }\end{array}$ & $\begin{array}{l}\text { Stomach } \\
\text { Small bowel } \\
\text { Other or intraperitoneally } \\
\text { with unknown primary } \\
\text { origin }\end{array}$ & $\begin{array}{c}16(27) \\
38(63) \\
6(10)\end{array}$ \\
\hline $\begin{array}{l}\text { Time on imatinib } \\
\text { therapy }\end{array}$ & $\begin{array}{l}\leq 12 \text { months } \\
>12 \text { months }\end{array}$ & $\begin{array}{l}12(20) \\
48(80)\end{array}$ \\
\hline Tumor genotype* & $\begin{array}{l}\text { Exon } 11 \text { KIT mutation } \\
\text { Exon } 9 \text { KIT mutation } \\
\text { PDGFRA mutation } \\
\text { Wild-type } \\
\text { Data not available }\end{array}$ & $\begin{array}{c}23(53.5) \\
11(25.6) \\
1(2.3) \\
8(18.6) \\
17\end{array}$ \\
\hline
\end{tabular}

cording to Response Evaluation Criteria in Solid Tumors (RECIST) version 1.1 [14]. In case of progression, patients were treated with other different tyrosine kinase inhibitors or cytotoxic chemotherapy or best supportive care only. If possible, they were included into clinical trials with new compounds. Toxic effects were graded with Common Terminology Criteria for Adverse Events (CTCAE), version 4.0.

\section{Genotyping}

Genomic screening was performed for the presence of the KIT (exons 9, 11, 13, and 17) or PDGFRA (exons 12, 14 and 18 ) genes mutation in 43 cases with fully available primary tumor specimens, based on DNA isolated from formalin-fixed paraffin-embedded or fresh frozen imatinib-naive tumor tissues, as previously described [4].

\section{Statistical analysis}

Contingency tables were analyzed by the chi-square test. Progression-free survival (PFS) time was calculated from the date of the start of sorafenib treatment to the date of the most recent follow-up, or progression or death due to the disease. Overall survival (OS) time was calculated either from the date of the start of imatinib and sorafenib treatment to the date of the most recent follow-up or death due to the disease. PFS/OS was assessed with respect to the following variables: demographic data (age at the diagnosis $\leq 45$ or $>45$ years; gender), primary tumor genotype (KIT 11 exon, KIT 9 exon, any PDGFRA mutations/wild-type cases), length of previous therapy on imatinib ( $\leq 12,>12$ months). The Kaplan-Meier method was used for analysis of survival curves, with log-rank test for univariate comparison of the survival between groups. Differences were considered statistically significant if $p$-values were $<0.05$. These statistical computations were performed using Statistica 7.1 software [Statsoft ${ }^{\circledR}$; Tulsa, OK].

\section{Results}

\section{Clinicopathological data}

The distribution of clinical and pathological data of patients included in the study is listed in Table 1. There were 40 male and 20 female patients, with median age at the start of sorafenib therapy 59 years. The majority of primary tumors were located in the small intestine (63\%), followed by the stomach (27\%). All patients had documented progression on imatinib and sunitinib, four patients had also received $3^{\text {rd }}$ line therapy before initiation of sorafenib - two with nilotinib and two with regorafenib. Majority of patients (80\%) were pre-treated with imatinib for more than one year. Almost $90 \%$ of patients started sorafenib therapy being in relatively good performance status (0-1).

\section{Mutational analysis data}

The distribution of patients according to the initial tumor mutational status is shown in Table 1 . In the group of 43 patients, whose initial tumor mutational status was evaluated, 54\% of GISTs had an exon 11 KIT mutation, 26\% had an exon 9 KIT mutation, 2\% had PDGFRA gene muta- 
tion and in $18 \%$ of tumors we have not detected any mutations (wild-type).

\section{Treatment toxicity}

Adverse events were common during sorafenib treatment (59/60 evaluated patients; $98 \%$ ), and in $35 \%$ of patients (21/60) they were assessed as grade 3/4 (Table 2), including one pancreatitis, two myocardial infarct, one DIC and two venous thrombosis. The most common non-hematological adverse events were: diarrhea, hand-foot syndrome (40\%), fatigue (65\%), and loss of weight (25.5\%). The frequency of reported laboratory tests and hematological toxicity was as follows: anemia (14\%), liver function test disturbances, hypocalcaemia and hypokalaemia (8\% each). One deaths (grade 5) due to pancreatitis and septic shock were assessed as related to reaction to sorafenib therapy. Twenty three patients required dose reduction of sorafenib (38\%), but only one patient had discontinued drug permanently due to toxicity.

\section{Outcomes of sorafenib treatment}

Median follow-up time from start of sorafenib therapy was 39 months. Median PFS was 7.7 months and estimated 1-year PFS rate was 23\% (Fig. 1). Progression of disease during sorafenib therapy was observed in 53 cases (88\%). At the time of the analysis, 9 patients (15\%) were alive. Estimated one-year OS rate was 57\%, 2-year OS rate was $26 \%$ and median OS was 13.5 months when calculated from sorafenib start (Fig. 2). Estimated 5-year OS in this group of patients was $65 \%$ (when calculated from the date of imatinib start) and median OS -7 years.

The best responses observed during sorafenib therapy and estimated by CT imaging (two consecutive examinations) according to RECIST criteria were as follows: none complete responses (CRs), 3 (5\%) partial responses (PR), 31 (52\%) stable disease (SD) lasting at least four months, $26(43 \%)$ progressive disease (PD). Overall clinical benefit of sorafenib therapy (counted as the sum of CR, PR and SD rates) was $57 \%$.

We have not found any relationship between the primary tumor genotype $(p=0.4)$ and outcomes of sorafenib therapy as well as the duration of initial imatinib therapy

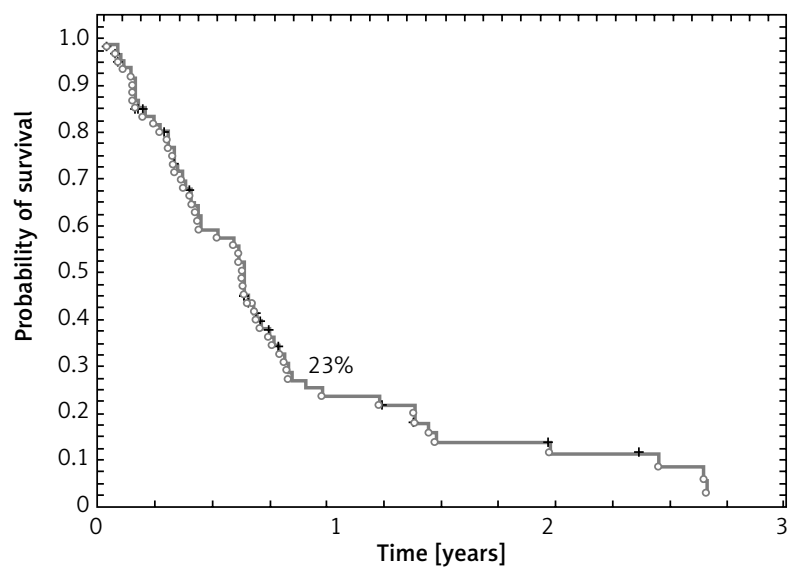

Fig. 1. Progression-free survival on sorafenib therapy
Table 2. The most common adverse events (AEs) during sorafenib therapy in the entire analyzed group of GIST patients

\begin{tabular}{lcccc|}
\hline & \multicolumn{2}{c}{ Any grade } & \multicolumn{2}{c}{ Grade $3 / 4$} \\
& $n$ & $\%$ & $n$ & $\%$ \\
\hline Any treatment-related AE & 59 & 98 & 21 & 35 \\
Diarrhea & 36 & 60 & 6 & 10 \\
Hand-foot syndrome & 34 & 57 & 8 & 13 \\
Fatigue & 21 & 35 & 1 & 2 \\
Loss of weight & 14 & 23 & 1 & 2 \\
Arterial hypertension & 11 & 18 & 0 & 0 \\
\hline Decreased appetite/dysgeusia & 11 & 18 & 0 & 0 \\
Skin reactions & 11 & 18 & 1 & 2 \\
\hline
\end{tabular}

( $p=0.3)$, patients' age $(p=0.7)$ or gender $(p=0.8)$ and survival on sorafenib therapy.

\section{Discussion}

In this study we have presented the largest one institution series of advanced GIST patients treated with sorafenib after failure of at least imatinib and sunitinib with the longest follow-up. The results of this study confirmed that significant proportion of advanced imatinib- and sunitinib-resistant GIST patients benefit from sorafenib therapy with median OS exceeding 1 year. Taking into account all limitations related to retrospective analysis these results in real-life setting seem to be comparable to outcomes of the $3 \mathrm{rd}$ line therapy with regorafenib (as presented in clinical trials) [6]. Sorafenib has been selected for therapy based on promising activity profile in in vitro studies against refractory GIST cell lines harboring secondary imatinib-resistant mutations [15-18]. Sorafenib is multikinase inhibitor approved for treatment of advanced renal cell carcinoma, hepatocellular carcinoma and thyroid carcinoma [19]. Sorafenib inhibits the activity of several intratumoral kinases (CRAF, BRAF, V600E BRAF, C-KIT, and FLT3) and targets in the tumor vasculature (CRAF, VEGFR-2, VEGFR-3, and PDGFR- $\beta$ ) [18]. Molecular mechanism activity of sorafenib in standard therapy refractory GIST is based on its ability for inhibition of many kinase KIT and

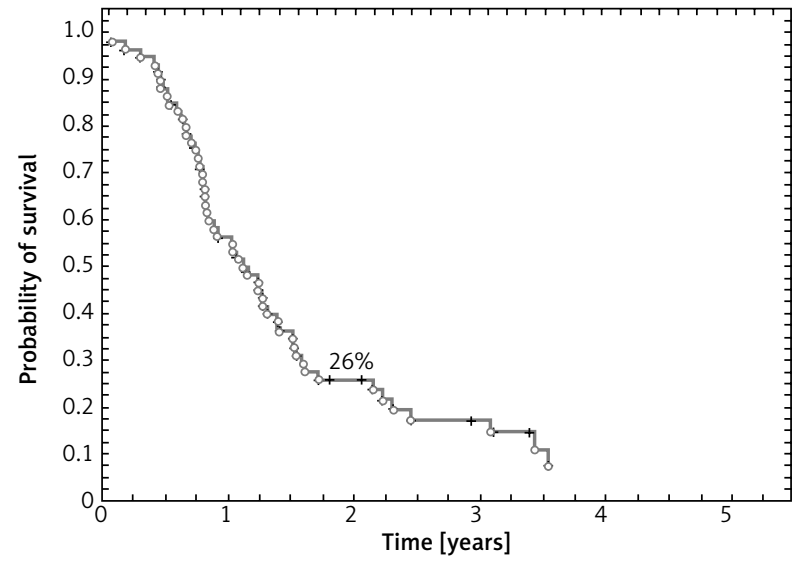

Fig. 2. Overall survival calculated from the date of start of sorafenib therapy 
PDGFRA mutations, which are imatinib-resistant and part of them also sunitinib-resistant. In in vitro studies Heinrich et al. determined that sorafenib inhibited imatinib-resistant mutations in exons encoding the ATP/drug-binding pocket (exon $11+13$ and exon $11+14$ ) and in exons encoding the activation loop (exon 17 and 18), with the exception of substitutions at KIT codon D816 and PDGFRA codon 842 [18]. Until now it has been studied in two small phase II trials and two retrospective series (smaller one center and larger multicenter with short follow-up) [10-12, 20, 21]. Two single-arm phase II clinical trials $(n=38$ and $n=31$ patients) have demonstrated activity in patients with GIST after progression to at least imatinib and sunitinib, with an clinical benefit rate exceeding $60 \%$ and disease control rate at 24 weeks $>30 \%$. Median PFS in both trials was approximately 5 months [10-12]. In a retrospective study of 223 imatinib- and sunitinib-resistant GIST patients, both nilotinib and sorafenib have significant clinical activity when given as a $3^{\text {rd }}$-line drug [22]. A retrospective analysis of sorafenib as $3^{\text {rd }}$ or $4^{\text {th }}$ line treatment in 124 patients with advanced GIST showed median PFS of 6.4 months and median OS identical to our study - 13.5 months [20]. Another multicenter study on 25 patients showed median PFS 7.2 months and median OS 15.2 months as the $3^{\text {rd }}$ - or

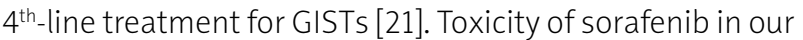
study was mostly manageable and comparable to those observed in other multikinase inhibitors $[5,6]$.

Other therapeutic options in refractory GIST are very limited - PAZOGIST study in GIST patients resistant to imatinib and sunitinib treated with pazopanib vs. best supportive care showed median PFS 3.4 months only in pazopanib arm. Several tyrosine kinase inhibitors as ponatinib, masitinib mesylate, BLU-285 and DCC-2618 are currently under investigations [22-27].

To the best of our knowledge, the present series represents the largest one center report with long-term follow-up concerning sorafenib use in imatinib- and sunitinib-resistant GIST. Our results, similar to previous studies, in heavily pretreated patients, showed the median PFS 7.7 months and median OS of 13.5 months on sorafenib therapy. Overall clinical benefit of sorafenib therapy was $57 \%$, what support the statement that sorafenib could be a reasonable treatment option in armamentarium in pretreated GIST patients with known safety profile although the toxicity was frequent and about one $3^{\text {rd }}$ of patients required dose reduction.

We confirmed that many advanced GIST patients benefit from sorafenib therapy after imatinib/sunitinib failure with OS > 1 year and we believe that sorafenib can be a substitute of regorafenib in the $3^{\text {rd }}$ line therapy of advanced GIST due to similar outcomes in real-life setting.

Piotr Rutkowski have received honoraria and was a member of Advisory Board for Novartis, he has received honoraria from lectures from Pfizer and he served as a member of Advisory Board for Bayer. The study has not received any external funding.

\section{References}

1. Joensuu H. Gastrointestinal stromal tumor (GIST). Ann Oncol 2006; 17 (Suppl 10): ×280-286.

2. Rutkowski P, Hompes D. Combined Therapy of Gastrointestinal Stromal Tumors. Surg Oncol Clin N Am 2016; 25: 735-59.

3. Demetri GD, von Mehren M, Blanke CD, et al. Efficacy and safety of imatinib mesylate in advanced gastrointestinal stromal tumors. New Engl J Med 2002; 347: 472-80.

4. Rutkowski P, Andrzejuk J, Bylina E, et al. What are the current outcomes of advanced gastrointestinal stromal tumors: who are the long-term survivors treated initially with imatinib? Med Oncol 2013; 30: 765

5. Reichardt P, Kang YK, Rutkowski P, et al. Clinical outcomes of patients with advanced gastrointestinal stromal tumors: safety and efficacy in a worldwide treatment-use trial of sunitinib. Cancer 2015; 121: 1405-13.

6. Demetri GD, Reichardt P, Kang YK, et al. Efficacy and safety of regorafenib for advanced gastrointestinal stromal tumours after failure of imatinib and sunitinib (GRID): an international, multicentre, randomised, placebo controlled, phase 3 trial. Lancet 2013; 381: 295-302.

7. Rutkowski P, Stępniak J. The safety of regorafenib for the treatment of gastrointestinal stromal tumors. Expert Opin Drug Saf 2016; 15: 105-16.

8. Wilhelm SM, Carter C, Tang L, et al. BAY 43-9006 exhibits broad spectrum oral antitumor activity and targets the RAF/MEK/ERK pathway and receptor tyrosine kinases involved in tumor progression and angiogenesis. Cancer Res 2004; 64(19): 7099-109.

9. Overton LC, Heinrich MC. Regorafenib for treatment of advanced gastrointestinal stromal tumors. Expert Opin Pharmacother 2014; 15: 549-58.

10. Wiebe L, Kasza KE, Maki RG, et al. Activity of sorafenib (SOR) in patients (pts) with imatinib (IM) and sunitinib (SU)-resistant (RES) gastrointestinal stromal tumors (GIST): a phase II trial of the University of Chicago Phase II Consortium. J Clin Oncol (Meeting Abstr) 2008; 26: 10502 .

11. Kindler HL, Campbell NP, Wroblewski K, et al. Sorafenib (SOR) in patients (pts) with imatinib (IM) and sunitinib (SU)-resistant (RES) gastrointestinal stromal tumors (GIST): final results of a University of Chicago Phase II Consortium trial. ASCO Meet Abstr 2011; 29: 10009.

12. Park SH, Ryu MH, Ryoo BY, Im SA, Kwon HC, Lee SS, Park SR, Kang BY, Kang YK. Sorafenib in patients with metastatic gastrointestinal stromal tumors who failed two or more prior tyrosine kinase inhibitors: a phase II study of Korean gastrointestinal stromal tumors study group. Invest New Drugs. 2012; 30: 2377-83.

13. NCCN Clinical Practice Guidelines in Oncology (NCCN Guidelines). Soft Tissue Sarcoma. Version 2.2017. NCCN.org (access on-line on 2nd October 2017).

14. Eisenhauer EA, Therasse P, Bogaerts J, et al. New response evaluation criteria in solid tumours: revised RECIST guideline (version 1.1). Eur J Cancer 2009; 45: 228-47.

15. Heinrich MC, Carden R, Griffith D, et al. In vitro activity of sorafenib against imatinib- and sunitinib-resistant kinase mutations associated with drug-resistant GI stromal tumors. ASCO Meet Abstr 2009; 27: 10500

16. Huynh H, Lee JW, Chow PK, et al. Sorafenib induces growth suppression in mouse models of gastrointestinal stromal tumor. Mol Cancer Ther 2009; 8: 152-9.

17. Guo T, Agaram NP, Wong GC, et al. Sorafenib inhibits the imatinib-resistant KITT670I gatekeeper mutation in gastrointestinal stromal tumor. Clin Cancer Res 2007; 13: 4874-81.

18. Heinrich MC, Marino-Enriquez A, Presnell Aet al. Sorafenib inhibits many kinase mutations associated with drug-resistant gastrointestinal stromal tumors. Mol Cancer Ther 2012; 11: 1770-80.

19. Nexavar. Summary of product characteristics. http://www.ema. europa.eu/docs/en GB/document library/EPAR - Product Information/human/000690/WC500027704.pdf

20. Montemurro M, Gelderblom $\mathrm{H}$, Bitz U, et al. Sorafenib as third- or fourth-line treatment of advanced gastrointestinal stromal tu- 
mour and pretreatment including both imatinib and sunitinib, and nilotinib: A retrospective analysis. Eur J Cancer 2013; 49: 1027-31.

21. Kefeli U, Benekli M, Sevinc A, et al. Efficacy of sorafenib in patients with gastrointestinal stromal tumors in the third- or fourth-line treatment: A retrospective multicenter experience. Oncol Lett 2013; 6: 605-11.

22. Italiano A, Cioffi A, Coco P, et al. Patterns of care, prognosis, and survival in patients with metastatic gastrointestinal stromal tumors (GIST) refractory to first-line imatinib and second-line sunitinib. Ann Surg Oncol 2012; 19: 1551-9.

23. Mir O, Cropet C, Toulmonde M, et al. Pazopanib plus best sup portive care versus best supportive care alone in advanced gastrointestinal stromal tumours resistant to imatinib and sunitinib (PAZOGIST): a randomised, multicentre, open-label phase 2 trial. Lancet Oncol 2016; 17: 632-41.

24. Eberst L, Cropet C, Le Cesne A, et al. The off-label use of targeted therapies in sarcomas: the OUTC'S program. BMC Cancer 2014, 14: 870 .

25. Soria JC, Massard C, Magne N, et al. Phase 1 dose-escalation study of oral tyrosine kinase inhibitor masitinib in advanced and/or metastatic solid cancers. Eur J Cancer 2009; 45: 2333-41.

26. Heinrich MC, von Mehren M, Demetri GD, et al. Ponatinib efficacy and safety in patients (pts) with advanced gastrointestinal stromal tumors (GIST) after tyrosine kinase inhibitor (TKI) failure: Results from a phase 2 study. I Clin Oncol 2015; 33 (suppl; abstr 10535).

27. BLU-285, DCC-2618 Show Activity against GIST. Cancer Discov 2017; 7: 121-2.

\section{Address for correspondence}

\section{Piotr Rutkowski}

Maria Sklodowska-Curie Institute - Oncology Center

Roentgena 5

02-781 Warsaw, Poland

e-mail: piotr.rutkowski@coi.pl

Submitted: 6.10 .2017

Accepted: 23.10.2017 